\title{
Risk of Ischemic Heart Disease in Patients with Non-alcoholic Fatty Liver Disease
}

\author{
Mostafa Soliman Elkady ${ }^{1}$, Hatem Samir Abd El-Raouf ${ }^{1}$, \\ Hany Ragheb Elkholy ${ }^{1}$, Badawy Abdul Khalek Abdul Aziz', \\ Wael Ahmed Maklad ${ }^{2}$, Ahmed Abd El-Allah Turkey ${ }^{3}$, \\ Hayam Abd EL-Hameed El-sayed ${ }^{1}$ \\ ${ }^{1}$ Department of Hepatology, Gastroenterology, and Infectious Diseases, \\ Faculty of Medicine, Benha University,Egypt \\ ${ }^{2}$ Department of Cardiology, Benha Teaching Hospital. \\ ${ }^{3}$ Department of Radiology, Faculty of Medicine Benha University.
}

Corresponding Author Hayam Abd ElHameed El -sayed

E mail:

drhaya@yahoo.com

Key words:

Fatty liver-Ischemic

Heart disease

Metabolic syndrome
Background and study aim: Nonalcoholic fatty liver disease (NAFLD) has an increasing prevalence worldwide. It has also been closely associated with obesity and metabolic syndrome - two conditions known to be associated with ischemic heart disease (IHD). The aim of this study was to assess the association between NAFLD and ischemic heart disease.

Subjects and Methods: 140 patients with NAFLD and 70 non-NAFLD subjects were selected. Full history taking, clinical examination and laboratory tests including blood sugar, lipid profile and liver profile were done. Ultrasonography was performed to prove NAFLD while ECG and echocardiography were used for detection of myocardial ischemia.

Results: Of the NAFLD group, the frequency of mild, moderate and severe

\section{INTRODUCTION}

NAFLD has become the predominant cause of chronic liver disease in many parts of the world. An overall global prevalence of $25.24 \%$ was reported with the highest figures were derived from the Middle East $(31.79 \%)$ and South America (30.45\%) [1].

NAFLD is considered a multisystem disease affecting several extrahepatic organs and regulatory pathways [2]. The major focus of NAFLD-related diseases has involved chronic liver disease, cardiovascular diseases and type $2 \mathrm{DM}$. There is also emerging evidence that NAFLD is linked to other diseases such as sleep apnea,
NAFLD was $42.9 \%, 30 \%$ and $27.1 \%$ respectively. Subjects with NAFLD had a significantly higher BMI, waist circumference and weight compared to those of non NAFLD group ( $\mathrm{p}=0.014,0.0218$ and $<0.001$ respectively). Independent risk factors for NAFLD were obesity, DM, high LDL, low HDL, waist circumference, glycated hemoglobin and IHD with odds ratios $1.09,2.12,1.01,1.15,1.13,1.37$ and 1.17 respectively. While independent risk factors for IHD included obesity, DM, high LDL, total cholesterol, triglycerides and the presence of NAFLD with odds ratios $1.31,1.23,1.19,1.132,1.68$ respectively.

Conclusion: NAFLD was independently associated with increased risk of myocardial ischemia.

colorectal cancer, osteoporosis, psoriasis and polycystic ovary syndrome [3].

Liver biopsy is considered the gold standard for diagnosing steatosis, but it is an invasive method associated with many adverse effects. Since US is relatively precise for the diagnosis of NAFLD, low-cost, risk-free and widely available, it has been a frequently used method [4]. Magnetic resonance imaging either by spectroscopy or by proton density fat fraction is an excellent noninvasive quantitative modality for assessment of steatosis and is being widely used in NAFLD clinical trials [5]. 
Patients with NAFLD have been shown to have increased mortality. The main cause of morbidity and mortality in these patients was cardiovascular disease [6]. Several studies have linked fatty liver to ischemic heart disease but they present controversial results. Some demonstrated an increased cardiovascular risk in NAFLD while others argued against this relationship. So, the role of NAFLD as an independent cardiovascular risk factor is still debated [7].

\section{The aim:}

To assess the possible relationship between nonalcoholic fatty liver disease and its associated risk factors on one hand and ischemic heart disease on the other hand.

\section{SUBJECTS AND METHODS}

\section{Subjects :}

The study was conducted on 210 patients (140 patients with ultrasonography-proven NAFLD and 70 subjects with no evidence of NAFLD serving as controls) attending Benha Teaching Hospital and Benha university hospital during the period from March 2016 to April 2017. All patients informed about the research and asked for their permission and consent before enrollment in the study.

\section{Inclusion criteria:}

1. Patients of both sexes were included.

2. Age: $18-65$.

3. Ultrasonography- proven NAFLD.

\section{Exclusion criteria:}

1. Advanced liver disease.

2. Hepatocellular carcinoma.

3. Comorbid liver disease and life threatening illness.

All Patients were subjected to thorough history taking, clinical examination including body mass index, weight and waist circumference and laboratory assessment including aspartate aminotransferase (AST), alanine aminotransferase (ALT), total \& direct bilirubin, total serum cholesterol, serum triglycerides (TG), serum high-density lipoprotein (HDL) cholesterol, fasting glucose, HbA1C, hepatitis B surface antigen, and an antibody to hepatitis $C$ virus.

Diagnosis of NAFLD: abdominal ultrasonography performed by an experienced radiologist was used for diagnosis of fatty liver. The criteria used were: increased echogenicity relative to the kidney, distal attenuation and loss of intra-hepatic architectural details. Fatty liver was classified according to the following items: echogenicity relative to kidneys (0-3), blurring of gall bladder wall (0-3), blurring of hepatic veins (0-3), blurring of portal vein (0-3), far gain attenuation $(0-3)$. Grading was defined as mild (total scores of 2-6), moderate (7-10), and severe (11-15) fatty liver [8]. Diagnosis of NALFD was done following exclusion of significant alcohol intake, other causes of chronic liver disease and use of drugs that could cause fatty liver.

Diagnosis of IHD: Was done using a combination of electrocardiography (ECG) and echocardiography.

\section{Statistical methods:}

The collected data were analyzed using SPSS software (statistical package for social science) version 22.0 on IBM compatible computer. Continuous variables were expressed as mean \pm standard deviation $(\mathrm{M} \pm \mathrm{SD})$, and analyzed using Student's $t$-test. Categorical variables were expressed as number and percentage and analyzed using Chi-square test or Fisher's exact test. Multivariate logistic regression analysis was used. Variables found to be significant on the univariate analysis to identify independent factors associated with NAFLD and IHD. The level of significance was set at a $P$-value of $<0.05$.

\section{RESULTS}

A total number of 210 participants were selected and divided into two groups; NAFLD group included 140 patients with ultrasonographyproven NAFLD ( 77 males \& 63 females) with mean age $45.81 \pm 12.46$ years and non-NAFLD group of 70 subjects with mean age 45.32 \pm 10.56 years. Of the NAFLD group, the frequency of mild, moderate and severe NAFLD was $42.9 \%$, $30 \%$ and $27.1 \%$ respectively. Subjects with NAFLD had a significantly higher BMI, waist circumference and weight compared to those of non NAFLD group $(\mathrm{p}=0.014,0.0218$ and $<0.001$ respectively). On the other hand, no significant difference was found between the two groups regarding the age, sex or height.

Regarding the evidence of myocardial ischemia, a statistically significant difference was found between NAFLD and non NAFLD groups with respect to the presence wall motion abnormalities 
and ischemic changes in ECG as shown in (Table 2). There was also a highly statistical significant difference among different grades of NAFLD $(\mathrm{p}<0.001)$.

On multivariate logistic regression analysis, independent risk factors for NAFLD were obesity, DM, high LDL, low HDL, waist circumference, glycated hemoglobin and IHD with odds ratios $1.09,2.12,1.01,1.15,1.13,1.37$ and 1.17 respectively (Table 3 ). While independent risk factors for IHD included obesity, DM, high LDL, total cholesterol, triglycerides and the presence of NAFLD with odds ratios 1.31, 1.23, 1.19, $1.13,1.68$ respectively (Table 4). Both IHD and NAFLD shared common risk factors such as obesity, presence of diabetes and dyslipidemia (increased LDL, low HDL). The risk of IHD was also significantly more in higher grades of NAFLD. The odds ratios for the different grades of NAFLD were; $1.56,2.11$ and 2.61 , for mild, moderate and severe NAFLD respectively.

Table (1): Clinical and laboratory characteristics of NAFLD and non-NAFLD groups

\begin{tabular}{|c|c|c|c|c|}
\hline & \multirow{2}{*}{$\begin{array}{c}\text { NAFLD } \\
\text { No (\%) }\end{array}$} & \multirow{2}{*}{$\begin{array}{c}\text { Non NAFLD } \\
\text { No }(\%) \\
\end{array}$} & \multicolumn{2}{|c|}{ Chi-square/ $t$-test } \\
\hline & & & $\mathbf{t} / \mathbf{X}^{2}$ & P-value \\
\hline \multicolumn{5}{|l|}{ Sex } \\
\hline Female & $63(45 \%)$ & $31(44.29 \%)$ & \multirow{2}{*}{0.10} & \multirow{2}{*}{0.922} \\
\hline Male & $77(55 \%)$ & $39(55.71 \%)$ & & \\
\hline Smoking & $51(36.4 \%)$ & $20(28.6 \%)$ & 1.287 & 0.257 \\
\hline \multicolumn{5}{|l|}{ BMI } \\
\hline Normal & $24(17.1 \%)$ & $13(18.6 \%)$ & \multirow{3}{*}{6.802} & \multirow{3}{*}{$0.033^{*}$} \\
\hline Over weight & $41(29.3 \%)$ & $32(45.7 \%)$ & & \\
\hline Obese & $75(53.6 \%)$ & $25(35.7 \%)$ & & \\
\hline \multirow[t]{2}{*}{$\overline{D M}$} & $90(64.3 \%)$ & $13(18.5 \%)$ & 58.372 & $<0.001 * *$ \\
\hline & Mean \pm SD & Mean \pm SD & $\mathbf{t} / \mathbf{X}^{2}$ & P-value \\
\hline Age (year) & $48.81 \pm 12.46$ & $49.32 \pm 10.56$ & & \\
\hline Height (cm) & $159.0 \pm 7.4$ & $161.4 \pm 8.4$ & 25.146 & 0.321 \\
\hline Weight (kg) & $75.5 \pm 10.0$ & $65.9 \pm 8.2$ & 9.422 & $<0.001 * *$ \\
\hline Waist circumference (cm) & $96.5 \pm 11.4$ & $90.3 \pm 12.9$ & 11.393 & $0.0218^{*}$ \\
\hline BMI $(\mathrm{kg} / \mathrm{m} 2)$ & $31.81 \pm 5.23$ & $25.4 \pm 3.45$ & 4.713 & $0.014 *$ \\
\hline HDL (mg/dL) & $38.4 \pm 12.1$ & $48.2 \pm 12.2$ & 3.487 & $0.004 *$ \\
\hline LDL $(\mathrm{mg} / \mathrm{dL})$ & $125.5 \pm 32.3$ & $104.3 \pm 24.1$ & 5.461 & $0.013 *$ \\
\hline Total cholesterol $(\mathrm{mg} / \mathrm{dL})$ & $215.6 \pm 39.1$ & $177.1 \pm 30.8$ & 3.730 & $0.009 *$ \\
\hline Serum triglycerides (mg/dL) & $179.7 \pm 64.7$ & $129.9 \pm 52.8$ & 3.256 & $0.012 *$ \\
\hline
\end{tabular}


Table (2): ECG and echocardiographic findings in different grades of NAFLD

\begin{tabular}{|c|c|c|c|c|c|c|}
\hline & \multicolumn{6}{|c|}{ Grades of NAFLD } \\
\hline & Non & Mild & Moderate & Severe & \multicolumn{2}{|c|}{ Chi-square } \\
\hline & $\mathbf{N}(\%)$ & $\mathbf{N}(\%)$ & $\mathbf{N}(\%)$ & $\mathbf{N}(\%)$ & $\mathbf{X}^{2}$ & P-value \\
\hline \multicolumn{7}{|c|}{ Wall motion abnormalities } \\
\hline Negative & $63(90 \%)$ & $39(84.8 \%)$ & $32(76.2 \%)$ & $39(75 \%)$ & \multirow{2}{*}{50.066} & \multirow{2}{*}{$<0.001 * *$} \\
\hline Positive & $7(10.00 \%)$ & $7(15.2 \%)$ & $10(23.8 \%)$ & $13(25 \%)$ & & \\
\hline \multicolumn{7}{|c|}{ ECG changes } \\
\hline Negative & $61(87.2 \%)$ & $38(82.6 \%)$ & $31(73.8 \%)$ & $35(67.3 \%)$ & \multirow{2}{*}{57.875} & \multirow{2}{*}{$<0.001 * *$} \\
\hline Positive & $9(12.8 \%)$ & $8(17.4 \%)$ & $11(26.2 \%)$ & $17(32.7 \%)$ & & \\
\hline
\end{tabular}

Table (3): Multivariate logistic regression analysis of the risk factors for NAFLD

\begin{tabular}{|l|c|c|c|c|}
\hline \multirow{2}{*}{ Obesity } & \multicolumn{2}{|c|}{ 95\% Confidence Interval } & \multirow{2}{*}{ Odds ratio } & \multirow{2}{*}{ P-value } \\
\cline { 2 - 3 } & Lower & Upper & & \\
\hline DM & 0.527 & 1.237 & 1.09 & $0.004^{*}$ \\
\hline IHD & 0.875 & 3.547 & 2.12 & $<0.001^{* *}$ \\
\hline Age & 0.943 & 2.165 & 1.17 & $0.005^{*}$ \\
\hline Waist circumference & 0.087 & 0.271 & 0.16 & 0.252 \\
\hline LDL & 1.00 & 1.15 & 1.13 & $0.019^{*}$ \\
\hline HDL & 1.00 & 1.18 & 1.01 & $0.022^{*}$ \\
\hline Total Cholesterol & 0.109 & 1.844 & 1.15 & $0.024^{*}$ \\
\hline S. Triglycerides & 0.498 & 0.977 & 0.52 & 0.156 \\
\hline HBA1C & 0.261 & 0.843 & 0.40 & 0.092 \\
\hline
\end{tabular}

Table (4): Multivariate logistic regression analysis of the risk factors for IHD

\begin{tabular}{|l|c|c|c|c|}
\hline \multirow{2}{*}{} & 95\% Confidence Interval & \multirow{2}{*}{ Odds ratio } & \multirow{2}{*}{ P value } \\
\cline { 2 - 5 } & Lower & Upper & 1.195 & $0.035^{*}$ \\
\hline Obesity & 0.870 & 1.275 & 1.234 & $0.032^{*}$ \\
\hline NAFLD: & 0.932 & 2.085 & 1.685 & $0.023^{*}$ \\
Mild. & 1.051 & 2.754 & 1.56 & $0.021^{*}$ \\
Moderate. & 1.071 & 2.524 & 2.11 & $0.016^{*}$ \\
Severe. & 1.356 & 3.341 & 2.61 & $0.011^{*}$ \\
\hline Age & 1.421 & 3.396 & 0.105 & 0.373 \\
\hline Waist circumference & 0.068 & 0.573 & 0.211 & 0.089 \\
\hline HDL & 0.132 & 0.506 & 0.058 & 0.458 \\
\hline LDL & 0.256 & 0.834 & 1.132 & $0.004^{*}$ \\
\hline Total Cholesterol & 0.99 & 1.537 & 1.31 & $0.010^{*}$ \\
\hline S. Triglycerides & 1.012 & 1.732 & 1.01 & 0.053 \\
\hline HBA1C & 1.003 & 1.089 & 0.514 & 0.654 \\
\hline
\end{tabular}




\section{DISCUSSION}

Metabolic syndrome features are not only highly prevalent in NAFLD patients, but components of metabolic syndrome also increase the risk of developing NAFLD [9]. There is a growing body of evidence that NAFLD and its risk factors carry an increased risk of cardiovascular disease independent of traditional cardiovascular risk factors and metabolic syndrome [10]. The most common cause of mortality in patients with NAFLD was presumed to be myocardial ischemia irrespective of other co-morbidities [11]. The possible contributing mechanisms are complex and heterogeneous including genetic predisposition, insulin resistance and dyslipidemia, chronic inflammation, oxidative stress, adiponectin deficiency and altered production of pro- and anticoagulant factors [12].

The current study aimed to investigate the association ultrasonography-diagnosed NAFLD and risk of IHD diagnosed by ECG and myocardial motion abnormality by echocardiography. In this study NAFLD group had a significantly higher BMI and waist circumference than the non NAFLD group. This was also observed by Loomis et al. who reported a 5- to 10-fold increased risk of NAFLD in the obese and 10- to 14-fold risk in the morbidly obese subjects. They concluded that both prevention of weight gain and weight reduction are important for prevention and management of NAFLD [13]. This finding also agrees with what reported by Sasaki et al. who observed that all grades of obesity are associated with NAFLD [14].

In the current study, there was a statistically significant positive association between diabetes mellitus and NAFLD as the diabetic patients had an odds ratio of 2.12 regarding the risk of developing NAFLD as revealed on multivariate analysis. This result agrees with several studies reported a high prevalence of NAFLD in patients with diabetes mellitus and a mutual association between NAFLD and DM [15,16]. Our study showed also a highly statistical significant difference between NAFLD and non-NAFLD groups as regarding the frequency of dyslipidemia. Patients with NAFLD had significantly higher levels of LDL cholesterol and triglycerides and lower levels HDL cholesterol than those of non-NAFLD group. Similar to our study Alper et al., Sun et al. and Kim et al. illustrated that high levels of triglycerides, LDL-cholesterol were significantly associated with increased risk of IHD [17-19].
Regarding the relationship between NAFLD and IHD, our study revealed a significantly positive association between degree of NAFLD diagnosed by ultrasonography and presence of ischemic heart disease. Finding was documented on both the univariate and the multivariate logistic regression analysis. The odds ratios for the various grades of NAFLD versus non- NAFLD group were 1.56, 2.11 and 2.61 for mild, moderate and severe NAFLD respectively. These data coincide with Pais et al. and Francque et al. who reported that there was a significant positive association between NAFLD and atherosclerotic process. They concluded that NAFLD represents an independent risk factor for coronary vascular disease [20, 21]. Our results also come in accordance with $\mathbf{Z i b}$ et $\boldsymbol{a l}$., 2016 in their prospective study on 4119 subjects after exclusion of coronary vascular disease or any known liver disease at baseline with a mean period of 7.6 years follow up [12]. They concluded that NAFLD was independently associated with the risk of adverse cardiovascular events. Furthermore, in a cross-sectional study on diabetic patients, Targher et al. found ultrasonography-diagnosed NAFLD to be associated with prevalent cardiovascular disease independent of classical risk factors, glycemic control, medications and metabolic syndrome features [22]. On the other hand, Chan et al. in their cross-sectional study on Asian diabetic patients reported that independent factors associated with IHD were older age, greater waist circumference, lower levels of physical activity and higher levels of HbAlc. They concluded that ultrasonography-diagnosed NAFLD was not associated with IHD. They explained this discrepancy in results by the differences in the study populations [23]. Pickhardt et al. also stated that steatosis was not found to be an independent risk factor for cardiovascular events on multivariate logistic regression analysis after controlling for other confounding risk factors [24].

Our study also showed that both NAFLD and IHD share common risk factors such as increased BMI, presence of diabetes and dyslipidemia. This result agree with what revealed by Lin et al. and Corey et al. who declared that the higher rates of IHD risk are associated with factors including hypertension, dyslipidemia, hyperglycemia and overweight. They also reported that IHD risk increased with the severity of fatty liver. They explained that patients with NAFLD have pro atherogenic lipid profile due to hepatic lipid concentration and insulin resistance $[\mathbf{2 5 , 2 6}]$. 
Some limitations of our study warrant to be mentioned. First, NAFLD patients in our study were not histologically diagnosed by biopsy. We diagnosed NAFLD using transabdominal ultrasonography that does not identify fatty infiltration $<30 \%$ and subjected to intra-and inter observer differences when making a diagnosis. However, it is a safe, available, of low cost, reasonably reliable and noninvasive method. To overcome this point, ultrasonography was performed by a single experienced operator. Second, assessment of ischemic heart did not depend on coronary angiography which is the gold standard but we used ECG and echocardiography instead. The usage of these non-invasive methods for diagnosing and grading NAFLD and also for detection of IHD risk have acceptable reliability and are practical tools of screening purpose [8]. In addition, this was not a population-based study covering a wide geographic area or a large number of patients. So our results needs to be furtherly evaluated for validation on larger cohorts.

\section{CONCLUSION:}

The current study revealed that presence of NAFLD is associated with increased risk of IHD. This association was not only due to common risk factors shared between the two entities such as obesity, dyslipidemia and diabetes, but also a direct independent positive relationship was found between them. So NAFLD can be considered as an independent predictor of myocardial ischemia.

\section{ACKNOWLEDGEMENT:}

The authors would like to acknowledge the help from all members of department of Hepatology, Gastroenterology and Infectious Diseases; department of Radiology and department of Cardiology in Benha University Hospital and Benha Teaching Hospital.

\section{REFERENCES}

1- Younossi ZM, Koenig AB, Abdelatif D, Fazel Y, Henry L,Wymer M. Global epidemiology of nonalcoholic fatty liver disease-Meta-analytic assessment of prevalence, incidence, and outcomes. Hepatology 2016; 64:73-84.

2- Masuoka HC, Chalasani N. Nonalcoholic fatty liver disease: an emerging threat to obese and diabetic individuals. Ann N Y Acad Sci 2014; 1281:106-122.
3- Byrne CD, Targher G. NAFLD: a multisystem disease. J Hepatol 2015; 62(1): S47-S64.

4- Bohte AE, van Werven JR, Bipat S, Stoker J. The diagnostic accuracy of US, CT, MRI and $1 \mathrm{H}-$ MRS for the evaluation of hepatic steatosis compared with liver biopsy: a meta-analysis. Eur Radiol 2011; 21:87-97.

5- Noureddin M, Lam J, Peterson MR, Middleton M, Hamilton G, Le TA, et al. Utility of magnetic resonance imaging versus histology for quantifying changes in liver fat in nonalcoholic fatty liver disease trials. Hepatology 2013; 58:1930-1940.

6- Francque SM, van der Graaff D, Kwanten WJ. Non-alcoholic fatty liver disease and cardiovascular risk: Pathophysiological mechanisms and implications. J Hepatol 2016; 65(2):425-43.

7- Ekstedt M, Hagström H, Nasr P, Fredrikson M, Stål P, Kechagias S, et al. Fibrosis stage is the strongest predictor for disease-specific mortality in NAFLD after up to 33 years of follow-up. Hepatology 2015; 61:1547-1554.

8- Saadeh S, Younossi ZM, Remer EM, Gramlich T, Ong JP, Hurley M, et al., The utility of radiological imaging in nonalcoholic fatty liver disease. Gastroenterology 2002; 123: 745-750.

9- Edens MA, Kuipers F, Stolk RP. Non-alcoholic fatty liver disease is associated with cardiovascular disease risk markers. Obes Rev 2009; 10:412-419.

10- Younossi Z, Henry L. Contribution of alcoholic and nonalcoholic fatty liver disease to the burden of liver related morbidity and mortality. Gastroenterology 2016; 150: 1778-1785.

11- Choi DH, Lee SJ, Kang CD, Park MO, Choi DW, Kim TS, et al. Nonalcoholic fatty liver disease is associated with coronary artery disease in Koreans. World J Gastroenterol 2013; 19(38):6453-7.

12- Zeb I, Li D, Budoff MJ, Katz R, Lloyd-Jones D, Agatston A, et al. Nonalcoholic Fatty Liver Disease and Incident Cardiac Events: The MultiEthnic Study of Atherosclerosis. J Am Coll Cardiol 2016; 67:1965-1966.

13- Loomis AK, Kabadi S, Preiss D, Hyde C, Bonato $\mathrm{V}$, St Louis M, et al. Body Mass Index and Risk of Nonalcoholic Fatty Liver Disease: Two Electronic Health Record Prospective Studies. J Clin Endocrinol Metab 2016; 101(3):945-52.

14- Sasaki A, Nitta H, Otsuka K, Umemura A, Baba $\mathrm{S}$, Obuchi T, et al. Bariatric surgery and nonalcoholic fatty liver disease: current and potential future treatments. Front Endocrinol (Lausanne) 2014; 5:164. 
15- Leite NC, Salles GF, Araujo AL, VillelaNogueira CA, Cardoso CR. Prevalence and associated factors of non-alcoholic fatty liver disease in patients with type 2 diabetes mellitus. Liver Int 2009; 29: 113-119.

16- Fruci B, Giuliano S, Mazza A, Malaguarnera R, Belfiore A.nonalcoholic fatty liver: a possible new target for type 2 diabetes prevention and treatment. Int J Mol Sci 2013; 14: 22933-66.

17- Alper AT, Hasdemir H, Sahin S, Ontürk E, Akyol A, Nurkalem $Z$, et al. The relationship between nonalcoholic fatty liver disease and the severity of coronary artery disease in patients with metabolic syndrome. Turk Kardiyol Dern Ars 2008; 36:376-81.

18- Sun L, Lu S . Association between non-alcoholic fatty liver disease and coronary artery disease severity. Chin Med J 2011; 124:867-72.

19- Kim D, Choi SY, Park EH, Lee W, Kang JH, Kim W, et al. Nonalcoholic fatty liver disease is associated with coronary artery calcification. Hepatology 2012; 56(2): 605-13.

20- Pais R, Giral P, Khan JF, Rosenbaum D, Housset C, Poynard T, et al. Fatty liver is an independent predictor of early carotid atherosclerosis. $J$ Hepatol; 65:95-102.

21- Francque SM, van der Graaff D, Kwanten WJ. Nonalcoholic fatty liver disease and cardiovascular risk: Pathophysiological mechanisms and implications. J Hepatol 2016; 65:425-443.

22- Targher G, Bertolini L, Padovani R, Poli F, Scala $\mathrm{L}$, Tessari $\mathrm{R}$, et al. Increased prevalence of cardiovascular disease in type 2 diabetic patients with non-alcoholic fatty liver disease. Diabet Med 2006; 23:403-9.

23- Chan WK, Tan AT, Vethakkan SR, Tah PC, Vijayananth A, Gohaa KL. Ultrasonographydiagnosed non-alcoholic fatty liver disease is not associated with prevalent ischemic heart disease among diabetics in a multiracial Asian hospital clinic population. Clin Res Hepatol Gastroenterol 2014; 38(3):284-91.

24- Pickhardt PJ, Hahn L, Muñoz del Rio A, Park SH, Reeder SB, Said A. Natural history of hepatic steatosis: observed outcomes for subsequent liver and cardiovascular complications. AJR Am J Roentgenol 2014; 202: 752-758.

25- Lin YC, Lo HM, Chen JD: Sonographic fatty liver, overweight and ischemic heart disease. World J Gastroenterol 2005; 11(31):4838-4842.

26- Corey KE, Misdraji J, Gelrud L, Zheng H, Chung RT, Krauss RM. Nonalcoholic steatohepatitis is associated with an atherogenic lipoprotein subfraction profile. Lipids Health Dis 2014; 13:100. 